\title{
Profiling the proteome-wide selectivity of diverse electrophiles
}

Patrick R. A. Zanon ${ }^{1}$, Fengchao Yu², Patricia Z. Musacchio ${ }^{3}$, Lisa Lewald ${ }^{1}$, Michael Zolloํ․ Kristina Krauskopf ${ }^{4}$, Dario Mrdović ${ }^{1}$, Patrick Raunft ${ }^{1}$, Thomas E. Maher ${ }^{1}$, Marko Cigler ${ }^{4}$, Christopher J. Chang ${ }^{5}$, Kathrin Lang ${ }^{4}$, F. Dean Toste ${ }^{3}$, Alexey I. Nesvizhskii ${ }^{2,6}$, and Stephan M. Hacker ${ }^{1, *}$

${ }^{1}$ Department of Chemistry, Technical University of Munich, Garching, Germany

2 Department of Pathology, University of Michigan, Ann Arbor, Michigan, USA

${ }^{3}$ Department of Chemistry, University of California, Berkeley, California, USA

${ }^{4}$ Department of Chemistry, Group of Synthetic Biochemistry, Technical University of Munich, Garching, Germany

${ }^{5}$ Departments of Chemistry and Molecular and Cell Biology, University of California, Berkeley, California, USA

${ }^{6}$ Department of Computational Medicine and Bioinformatics, University of Michigan, Ann Arbor, Michigan, USA

*Correspondence: stephan.m.hacker@tum.de

\begin{abstract}
Targeted covalent inhibitors are powerful entities in drug discovery, but their application has so far mainly been limited to addressing cysteine residues. The development of cysteine-directed covalent inhibitors has largely profited from determining their proteomewide selectivity using competitive residue-specific proteomics. Several probes have recently been described to monitor other amino acids using this technology and many more electrophiles exist to modify proteins. Nevertheless, a direct, proteome-wide comparison of the selectivity of diverse probes is still entirely missing. Here, we developed a completely unbiased workflow to analyse electrophile selectivity proteome-wide and applied it to directly compare 54 alkyne probes containing diverse reactive groups. In this way, we verified and newly identified probes to monitor a total of nine different amino acids as well as the $\mathrm{N}$-terminus proteome-wide. This selection includes the first probes to globally monitor tryptophans, histidines and arginines as well as novel tailored probes for methionines, aspartates and glutamates.
\end{abstract}

\section{Introduction}

Targeted covalent inhibitors (TCls) are powerful entities in drug discovery as they can have key advantages such as increased binding affinity to the target, the possibility to generate selectivity among closely related proteins and better pharmacodynamic properties. ${ }^{1}$ Nevertheless, careful optimisation of the reactivity and selectivity of these inhibitors is key to avoiding toxicity as well as possible immunogenic reactions. ${ }^{1}$

For TCls that target cysteine residues, competitive residue-specific proteomics is an essential tool to tailor covalent inhibitors for high selectivity proteome-wide. ${ }^{2,3}$ The underlying methods are based on the ground-breaking isotopic tandem orthogonal proteolysis activitybased protein profiling (isoTOP-ABPP) platform. ${ }^{2,3}$ We have recently built on this platform by developing isotopically labelled desthiobiotin azide (isoDTB) tags to streamline the experimental procedure. ${ }^{4}$ In the underlying isoDTB-ABPP workflow ${ }^{4}$ (Fig. 1a), two samples of a proteome-of-interest are treated with a covalent ligand or the corresponding solvent as a control. Next, a broadly reactive alkyne probe is applied that labels many residues with alkynes. The residue(s) that are already engaged by the ligand are blocked from this reactivity and are not modified with alkynes. In the next step, isotopically differentiated isoDTB tags are attached using coppercatalysed azide-alkyne cycloaddition (CuAAC). ${ }^{5}$ As the proteins originating from the compound- and solvent-treated samples are now
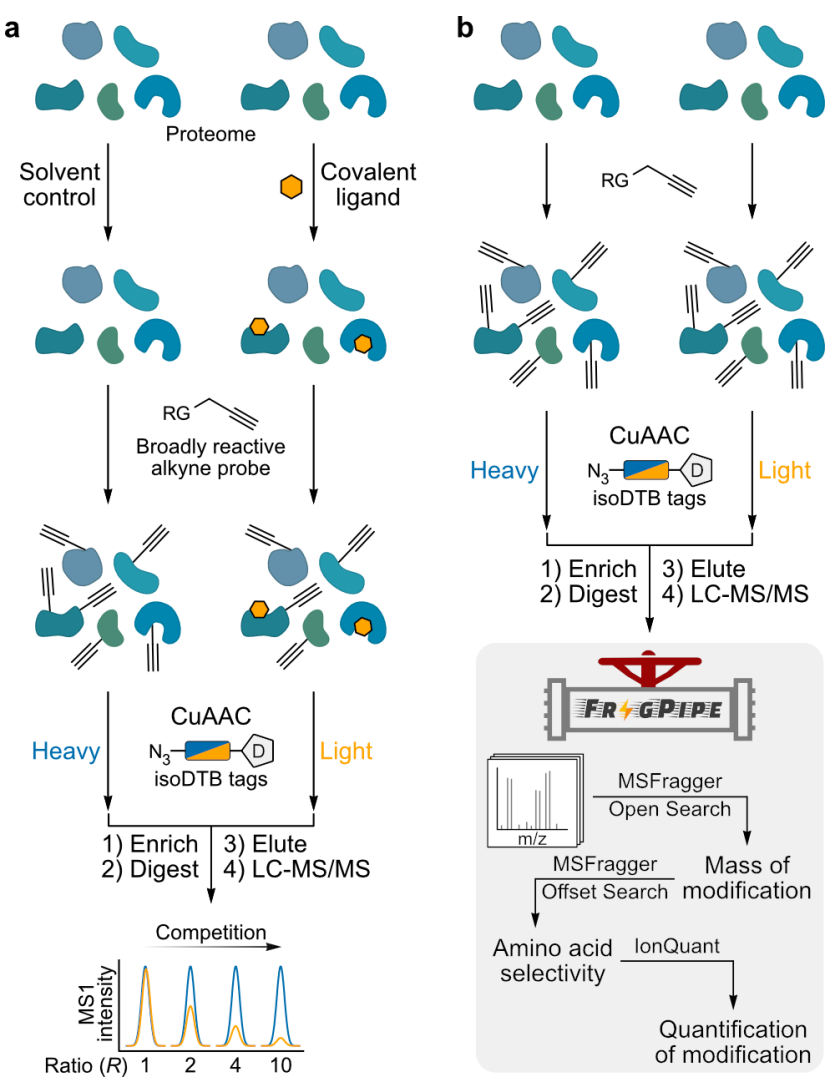

Fig. 1 | Workflows for competitive isoDTB-ABPP and the unbiased analysis of electrophile selectivity. a, Workflow for competitive, residue-specific chemoproteomic experiments using the isoDTB-ABPP workflow. ${ }^{4} \mathrm{RG}=$ reactive group; $\mathrm{D}=$ desthiobiotin. $\mathbf{b}$, Unbiased workflow to comprehensively investigate electrophile reactivity in the proteome using the MSFragger-based FragPipe computational platform.

differentiated, the two samples are mixed, enriched, proteolytically digested and the modified peptides eluted. Using liquid chromatography coupled to tandem mass spectrometry (LC-MS/MS), the modified peptides are identified and quantified. Peptides containing residues that are engaged by the covalent ligand will show high ratios between the two samples $(R » 1)$, while all unaffected peptides will show ratios close to 1 . In this way, target engagement and selectivity of $\mathrm{TCls}$ can be investigated proteome-wide in a quantitative fashion. ${ }^{4}$ 
a

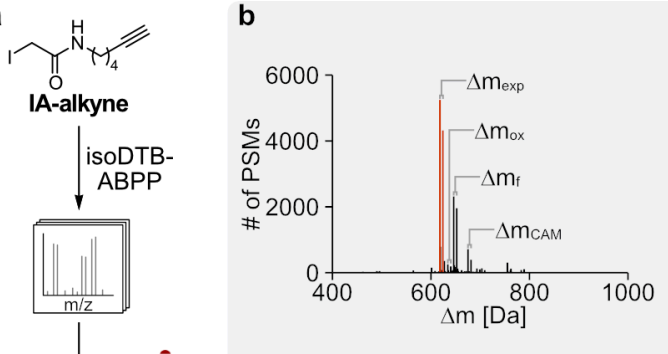

c

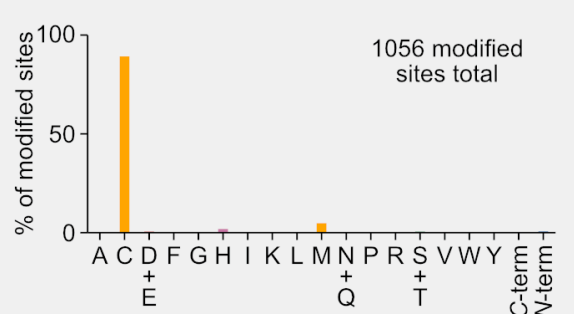

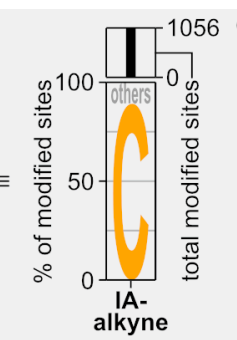

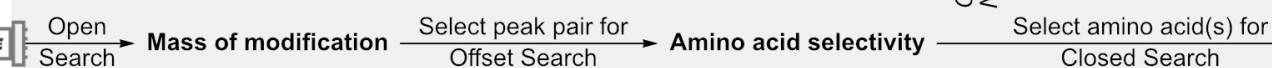

d Quantified sites

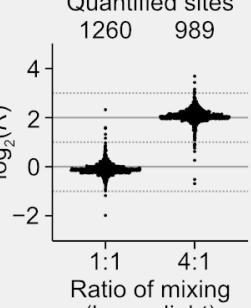

(heavy:light)

Quantification

Fig. 2 | An unbiased workflow to study electrophile selectivity using the MSFragger-based FragPipe computational platform. a, Labelling of the proteome of $S$. aureus SH1000 with $1 \mathrm{mM} \mathrm{IA-alkyne} \mathrm{and} \mathrm{analysis} \mathrm{using} \mathrm{the} \mathrm{isoDTB-ABPP} \mathrm{workflow} \mathrm{(Fig} 1 \mathrm{~b}$ ) ${ }^{4}$ results in MS data for the analysis of proteome-wide reactivity and selectivity. $\mathbf{b}$, Through analysis with an Open Search in MSFragger, ${ }^{6,7}$ the masses of modification that occur proteome-wide are assigned. The peaks highlighted in red are the expected modifications $\left(\Delta \mathrm{m}_{\mathrm{exp}}\right)$ resulting from alkylation and modification with the light and heavy isoDTB tags, respectively. Further modification of the alkylated peptides by oxidation ( $\left.\Delta \mathrm{m}_{\mathrm{ox}}\right)$, formylation $\left(\Delta \mathrm{m}_{\mathrm{f}}\right)$ or carbamidomethylation on a second cysteine $\left(\Delta \mathrm{m}_{\mathrm{CAM}}\right)$ is also detected. PSMs = peptide spectrum matches. c, One peak pair $\left(\Delta \mathrm{m}_{\text {exp }}\right)$ is selected for an MSFragger Offset Search that localises this modification to the modified amino acid(s). In this way, selectivity is assessed across all proteinogenic amino acids. The bar graph represents the fraction of all modified sites that is modified at the indicated amino acid. The same data is also represented in a letter plot, in which the size of the letter is scaled by the fraction of all modified sites that is modified at the indicated amino acid. All amino acids that are modified in less than $5 \%$ of the cases are summarised as "others". The total number of modified sites is given as a bar graph on top of the letter plot. $\mathbf{d}$, One amino acid (cysteine) is selected for quantification at the selected masses ( $\Delta \mathrm{m}_{\mathrm{exp}}$ ) using an MSFragger Closed Search and the lonQuant quantification module. ${ }^{8}$ Here, two datasets were analysed, in which the heavy and light samples were mixed at a ratio of $1: 1$ and $4: 1$, respectively. The grey, solid lines indicate the expected values of $\log _{2}(R)=0$ and $\log _{2}(R)=2$. The grey, dashed lines indicate the respective preferred window of quantification $\left(-1<\log _{2}(R)<1\right.$ for the $1: 1$ ratio, $1<\log _{2}(R)<3$ for the $4: 1$ ratio). The total number of quantified sites is given on top of the plot. The mass spectrometric experiments used for this analysis were performed in an earlier study. ${ }^{4}$ All data is based on technical duplicates.

Despite the promise of TCls to address diverse reactive amino acids, they so far almost exclusively address cysteine residues. ${ }^{9}$ However, as cysteine is a very rare amino acid, many interesting binding pockets contain no suitable cysteine for covalent engagement. ${ }^{10}$ Recently, many reactive groups have been developed that selectively target other residues in proteins and that have promise for $\mathrm{TCl}$ development against a much broader set of target proteins. ${ }^{11}$ For these compounds, one key challenge is to globally investigate their target and amino acid selectivity. Alkyne-, azide- or (desthio)biotin-containing probes have been successfully developed in various cases to directly investigate global selectivity. ${ }^{12}$ Nevertheless, besides often being synthetically challenging, this approach requires that the modification is stable at all modified residues, which excludes this strategy e.g. for acylating reagents that form unstable thioesters at cysteine, which evade direct detection. ${ }^{13}$ Similarly, this approach is not suitable for reversibly covalent inhibitors, ${ }^{14,15}$ whose modifications would also be lost before the analysis. In contrast, if one broadly reactive alkyne probe is developed that leads to a stable modification of an amino acid of interest, the targets for all of these compounds can be identified competitively. Therefore, being able to competitively profile target engagement at various amino acids using residue-specific proteomics would enable a global profile of the selectivity of TCls regardless of their amino acid selectivity or the stability of their adducts.

To date, broadly reactive alkyne probes that can be used in this approach have been reported for cysteines, ${ }^{2,3,16,17}$ lysines, ${ }^{18,19}$ aspartates and glutamates, ${ }^{10,20}$ methionines ${ }^{21,22}$ as well as tyrosines..$^{23,24}$ In the different underlying studies various strategies for the affinity tags, the isotopic labelling, the mass spectrometric instrumentation and the data analysis have been used. In this way, it is impossible to directly compare the reactivity and selectivity of these probes and to choose the best probe for a certain application. Furthermore, for other amino acids, broadly reactive alkyne probes are still entirely missing. While a direct comparison of the amino acid selectivity of five carbon electrophiles has been performed previously, ${ }^{25}$ a broad scale comparison of many diverse electrophiles is still entirely missing. To address this challenge, we here directly compared a large variety of electrophiles and established their proteome-wide amino acid selectivity using isoDTB-ABPP. ${ }^{4}$ This method is ideally suited to perform this analysis as it allows specific enrichment of the modified peptides to increase sensitivity of detection. Furthermore, it fosters confident assignment of probelabelled peptides due to the isotopic pattern introduced by the isoDTB tags.

As we are most interested in the application of TCls for antibacterial applications, ${ }^{4}$ we performed the main part of our analysis in the lysate of Staphylococcus aureus $\mathrm{SH} 1000 .^{26}$ We enhanced and developed new features for the MSFragger-based ${ }^{6,7}$ FragPipe computational platform (https://fragpipe.nesvilab.org), which now allows studying proteome-wide electrophile reactivity in a completely unbiased fashion (Fig. 1b). In this way, we verified or newly identified probes to competitively study a total of nine different amino acids as well as the $\mathrm{N}$-terminus proteome-wide. For the chosen amino acid-selective probes, we verify similar selectivity in the human proteome indicating that amino acid selectivity is not dependent on the specific proteome. This set of probes will enable competitive profiling of TCls not only against cysteine but against a variety of reactive amino acids and thereby guide the development of novel TCls.

\section{Results and Discussion}

Tailoring the MSFragger-based FragPipe computational platform for unbiased analysis of proteome-wide electrophile selectivity. One important challenge for the widespread application of competitive residue-specific proteomics is that tailored software is not readily available to the broader community. ${ }^{27}$ Furthermore, it was so far not possible to study electrophile reactivity and selectivity in a completely unbiased fashion. In order to address these challenges, we set out to tailor the MSFragger software and other components of the FragPipe computational platform for this application. ${ }^{6,7} \mathrm{We}$ chose to use FragPipe as it is freely available to the academic community and as the ultrafast fragment-ion indexing method is especially powerful for the complex data analyses needed to identify and localise modifications on peptides in an unbiased fashion. To validate the new software features, we utilised a published dataset, ${ }^{4}$ in which $1 \mathrm{mM}$ iodoacetamide alkyne (IA-alkyne) was used in a noncompetitive isoDTB-ABPP workflow (Fig. 1b). 


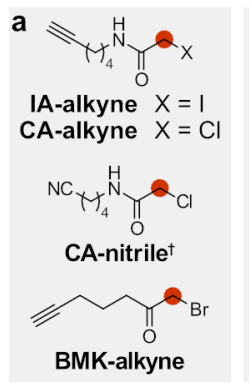

b
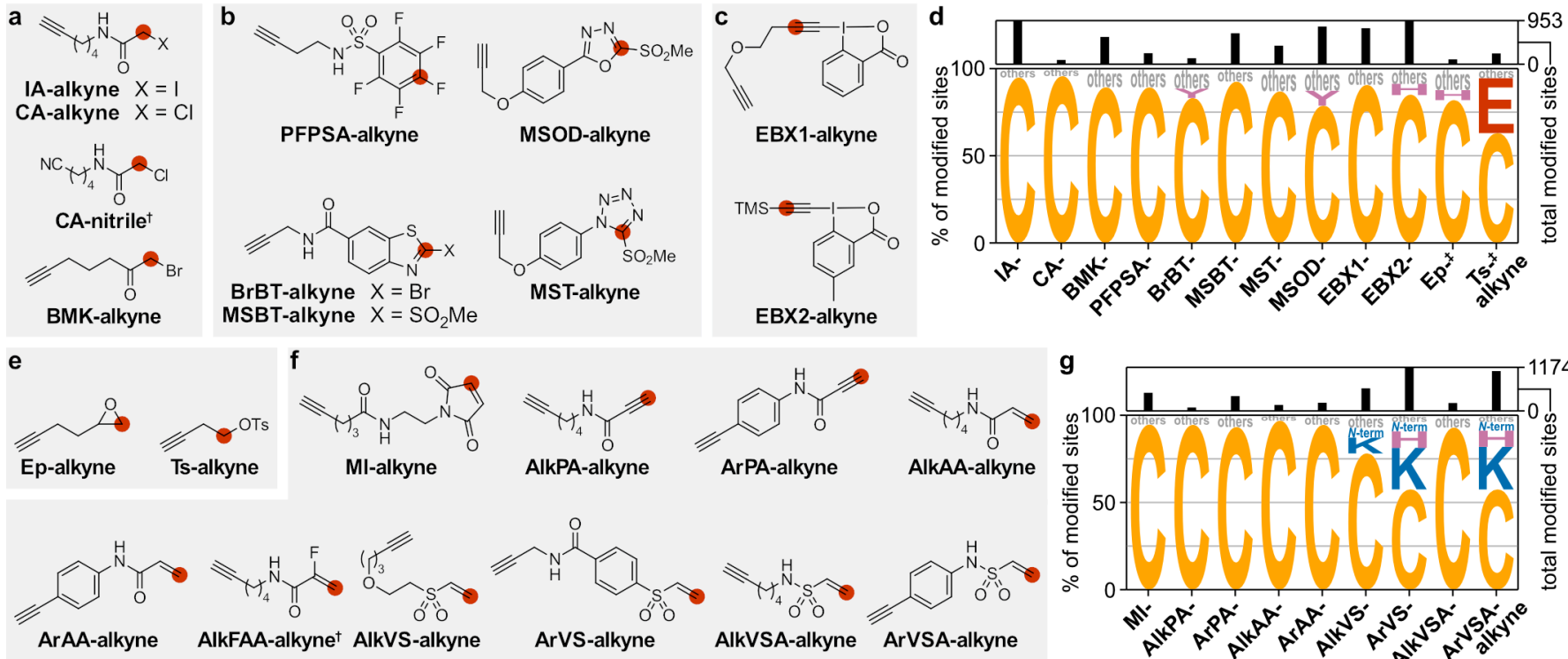

AlkPA-alkyne

ArPA-alkyne

AlkAA-alkyne

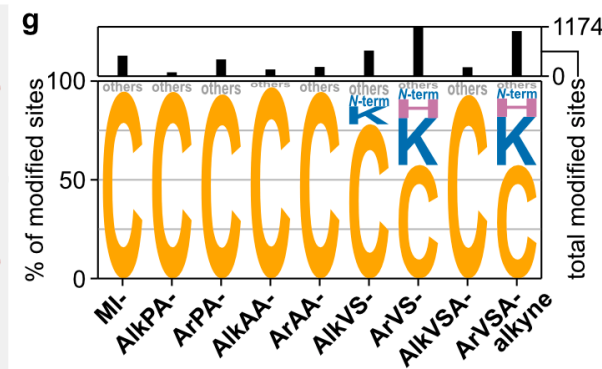

Fig. 3 | Amino acid selectivity of electrophiles targeting cysteines. a,b,c,e,f, Structures of alkyne-containing electrophilic probes that were investigated for their proteomewide amino acid selectivity. Red circles indicate the initial site of electrophilic reactivity. $\mathbf{d}, \mathbf{g}$, Amino acid selectivity of the probes upon treatment of the proteome of $S$. aureus $\mathrm{SH} 1000$ at $100 \mu \mathrm{M}$ probe concentration. The data is represented as letter plots. The size of the letter is scaled by the fraction of all modified sites that is modified at the indicated amino acid. All amino acids that are modified in less than $5 \%$ of the cases are summarised as "others". The total number of modified sites is given as a bar graph on top of the letter plot. ${ }^{\dagger}$ : No clear mass of modification was detected and therefore no analysis of the amino acid selectivity was possible. $\neq^{\prime}$. Data for the indicated probe at $1 \mathrm{mM}$ is shown. All data is based on technical duplicates.

In the first step, we optimised FragPipe's Open Search workflow to identify modified peptides and assign the masses of their respective modifications. For this purpose, we performed an Open Search using MSFragger ${ }^{6,7,28,29}$ followed by removal of mass shift artifacts with Crystal- ${ }^{28}{ }^{28}$ processing of peptide and protein identifications using PeptideProphet ${ }^{30}$ and ProteinProphet, ${ }^{31}$ false discovery-rate (FDR) filtering via Philosopher ${ }^{32}$ and summarisation of the mass shifts observed on peptides using PTM-Shepherd. ${ }^{29}$ Using this procedure, we identified the expected alkylation with IA-alkyne as the main modification $\left(\Delta \mathrm{m}_{\mathrm{exp}}\right)$ in the form of two peaks corresponding to the isotopically differentiated isoDTB tags (Fig. 2a,b). Furthermore, we detected a significant amount of additional formylation $\left(\Delta \mathrm{m}_{\mathrm{f}}\right)$ of the modified peptides, which is known to occur when eluting, re-dissolving and storing peptides in solutions containing formic acid. ${ }^{33}$ Additionally, we identified smaller peaks that correspond to oxidation of the formed thioether $\left(\Delta \mathrm{m}_{\mathrm{ox}}\right)$ or additional carbamidomethylation at a second cysteine residue $\left(\Delta \mathrm{m}_{\mathrm{CAM}}\right)$. The detection of these expected minor modifications verifies that MSFragger, with its ability to perform accurate deisotoping ${ }^{34}$ and mass calibration, ${ }^{7}$ can identify different modifications occurring after treatment with an electrophilic probe proteome-wide in an unbiased fashion with high mass accuracy. As the highest deviation of all of these masses of modification from the expected value was $0.0044 \mathrm{Da}(6.9 \mathrm{ppm})$, the molecular formula can be directly deduced from the MS data for unknown modifications.

After having determined the mass of the modification, we performed an MSFragger Offset Search, which allows searching at the mass with an indicated offset from the mass of the unmodified peptide. ${ }^{6,7}$ Here, we chose an offset that accounts for the detected alkylation with IA-alkyne $\left(\Delta \mathrm{m}_{\mathrm{exp}}\right)$. The modification is computationally localised to an amino acid residue or a stretch of amino acids without having to previously specify, which amino acids are potentially modified. ${ }^{7}$ Thus, the FragPipe computational platform allows to globally study selectivity towards all amino acid residues and the protein termini at once. After thorough data filtering, we verified that IA-alkyne shows high selectivity of $89 \%$ for cysteines with alkylation of methionines (5\%) being the most prominent additional reactivity (Fig. 2c).
Finally, to use probes for competitive residue-specific proteomics, their relative intensity between the light and the heavy channel needs to be quantified. For this purpose, complex in-house software ${ }^{3,35}$ or workarounds in existing software ${ }^{4}$ had to be used, so far. Therefore, we extended the quantification tool lonQuant ${ }^{8}$ - another FragPipe component - to allow quantification of modified peptides in MSFragger Offset Searches as well as in conventional Closed Searches, in which the modified residue is specified. To do so, we implemented a new functionality in lonQuant to perform relative quantification of isotopically labelled peaks to support stable isotope labelling-based quantification. In a Closed Search, we quantified 1260 cysteines with $>99 \%$ in the preferred quantification window of $-1<\log _{2}(R)<1$ (Fig. 2d). For the Offset Search, we quantified 1896 modified peptides $(99 \%$ in the preferred quantification window, Supplementary Fig. 1). It should be noted that the number of total modified sites, which is used for selectivity analysis based on an Offset Search (1056 total modified sites in this case, Fig. 2c), and the number of quantified residues in the Closed Search (1260 quantified cysteines in this case, Fig. 2d) or the Offset Search (1896 quantified peptides in this case, Supplementary Fig. 1) will be different in most cases, because of different data analysis workflows and downstream data filtering. We also analysed an additional published dataset, ${ }^{4}$ in which the heavy and light sample were mixed at a ratio of 4:1. Here, we quantified 989 cysteines with $98 \%$ in the preferred quantification window of $1<\log _{2}(R)<3$ (Fig. $2 \mathrm{~d}$ ). The high quality of this quantification data was comparable to data evaluation with MaxQuant ${ }^{36}$ using our previously described workaround ${ }^{4}$ or $p F i n d 3^{37}$ using a custom script for downstream analysis ${ }^{35}$ (Supplementary Fig. 2). Importantly, our automated FragPipe workflow simplifies the data analysis and allows analysis for probes that are not selective for a certain amino acid type.

Overall, the optimised FragPipe computational platform enables the completely unbiased analysis of residue-specific proteomic data obtained with various probes including identification of the mass of the modification, its amino acid selectivity and its use for quantitative applications. Having this unbiased analysis workflow at hand, we applied a standardised sample preparation protocol for all probes 
throughout this project to directly compare them regarding their proteome-wide reactivity and selectivity. Here, two identical samples of $S$. aureus lysate were treated with $100 \mu \mathrm{M}$ of the respective probe, modified with $100 \mu \mathrm{M}$ of the light or heavy isoDTB tag, respectively, mixed at a ratio of $1: 1$ and analysed using the isoDTB-ABPP workflow.

Diverse chemistries allow monitoring of cysteines. Using the described standard conditions for IA-alkyne ${ }^{3}$ (Fig. 3a), we detected 95\% selectivity for cysteines and quantified 1197 cysteines (Fig. 3d, Supplementary Fig. 3 and 4). An increase of the concentration to $1 \mathrm{mM}$ resulted in $86 \%$ selectivity for cysteines. Chloroacetamide CA-alkyne $^{3}$ and $\alpha$-bromomethyl ketone BMK-alkyne ${ }^{38}$ (Fig. 3a) also demonstrated high selectivity for cysteines (96\% and $89 \%)$ and allowed quantification of 230 and 976 cysteines, respectively (Fig. 3d, Supplementary Fig. 3 and 4). Importantly, a chloroacetamide negative control (CA-nitrile, Fig. 3a), with a nitrile replacing the alkyne, did not yield a clear mass of modification (Supplementary Fig. 3).

Nucleophilic aromatic substitution is an alternative chemistry to label cysteine residues in proteins (PFPSA-, ${ }^{39}$ BrBT-, MSBT-, MST- and MSOD-alkyne, ${ }^{16}$ Fig. $\left.3 b\right)$. All of the studied probes showed high cysteine selectivity (70-92\%) and allowed quantification of 362-1061 cysteines (Fig. 3d, Supplementary Fig. 5 and 6). We also investigated hypervalent iodine reagents that have been described for labelling of cysteines in the proteome. ${ }^{17}$ Using the originally reported chemistry (EBX1-alkyne, Fig. 3c, Supplementary Fig. 7), ${ }^{40}$ we detected several different modifications (Supplementary Fig. 8). ${ }^{41}$ While all of these showed high cysteine selectivity (91-97\%, Fig. 3d, Supplementary Fig. 8 and 9), this complex modification behaviour complicates downstream data analysis. Therefore, we turned to the recently published probe EBX2-alkyne ${ }^{42}$ (Fig. 3c), which selectively led to the minimal modification with an ethynyl group (Supplementary Fig. 8, 9 and 10). This modification showed high selectivity for cysteines (85\%) and allowed quantification of 1251 cysteines in the proteome (Fig. 3d, Supplementary Fig. 8 and 9). Finally, we also turned to nucleophilic substitution reactions at unactivated $\mathrm{sp}^{3}$-carbon centres (Ep- and Ts-alkyne, Fig. 3e). Both probes showed a clear preference for cysteines, but for Ts-alkyne a significant number of modifications was also found at glutamates (Fig. 3d, Supplementary Fig. 11 and 12).

When we combined the quantified cysteines of all these probes, we in aggregate quantified 1941 cysteines in S. aureus covering $37 \%$ of the 5268 cysteines encoded in the $S$. aureus genome. IA-alkyne can still be considered the gold standard for monitoring cysteine residues with residue-specific proteomics in vitro. Nevertheless, we verified that many other complementary probes exist that further increase the coverage with EBX2- and MSBT-alkyne being especially powerful (Supplementary Fig. 13).

Michael acceptors preferentially react with cysteines. Michael acceptors are currently mainstays for the design of TCls (Fig. 3f). For a maleimide probe (Ml-alkyne, after hydrolysis of the resulting succinimide during the workflow) and for propiolamide probes (AlkPA- and ArPA-alkyne), we detected high cysteine selectivity (93-95\%) and quantified a total of 283-752 cysteines (Fig. 3g, Supplementary Fig. 14 and 15). We also observed the expected modification and strong proteomic labelling (160-1174 localised sites, Fig. 3f,g, Supplementary Fig. 16-19) for all acceptorsubstituted terminal alkenes except for AlkFAA-alkyne. Strikingly, we observed different degrees of selectivity. While AlkAA-alkyne labelled cysteine residues with $97 \%$ selectivity, less than $60 \%$ of labelled sites were cysteines for ArVSA- and ArVS-alkyne (>20\% lysines, $\sim 9 \%$ histidines, $\sim 5 \% \mathrm{~N}$-termini). Interestingly, the labelling of
$N$-termini showed a preference for the secondary amine of proline when the initial methionine was removed (Supplementary Fig. 19). ${ }^{43}$ This data demonstrates that, even though Michael acceptors can be designed to also target other amino acid residues, cysteines are the major modification sites in all studied cases. In this way, while lysineselective TCls have been developed based on Michael acceptors, ${ }^{44}$ monitoring their cysteine off-targets is an important prerequisite for their further development.

Studying lysine residues proteome-wide. The activated ester probe STP-alkyne (Fig. 4a) allows selective monitoring of many lysine residues in the proteome. ${ }^{18}$ Using our workflow, we verified its high selectivity for lysines (78\%, Fig. 4b, Supplementary Fig. 20 and 21 ), which led to the quantification of 3277 lysines. The remaining peptides were mainly labelled at serines $(9 \%)$, threonines $(2 \%)$ or $\mathrm{N}$-termini (5\%). This selectivity was fully retained even at $1 \mathrm{mM}$ probe concentration. It is noteworthy that peptides labelled at threonine preferentially have a histidine at position -2 and those labelled at serine show a preference for cysteine at position +2 , arginine at position -1 and histidine at position -2 indicating that these sequence contexts might increase reactivity towards STP-alkyne (Supplementary Fig. 21). ${ }^{43}$ We also studied four additional acylation reagents (TFP-,${ }^{18}$ NHS-,${ }^{19}$ ATT- -45 and NASA-alkyne,${ }^{46}$ Fig. $4 a$ ). and detected an overall similar selectivity, which allowed quantification of 2145-4404 lysines (Fig. 4b, Supplementary Fig. 20 and 22). All of these probes are therefore suitable to study lysines proteome-wide and hold potential especially for applications that are hampered by the negative charge of STP-alkyne. All of the probes showed similar preferences in the sequence around labelled serines and threonines as seen for STP-alkyne (Supplementary Fig. 22). As there are no tools to globally study serines and threonines in a residue-specific fashion and as tools to study the $\mathrm{N}$-terminus lead to limited coverage (vide infra), it is furthermore noteworthy that STP-alkyne also quantified 428 serines, 165 threonines and $152 \mathrm{~N}$-termini. This points to the fact that, while STP-alkyne mainly labels lysines, it could also moonlight to detect effects on these other amino acids.

Besides acylation reagents, squaric acid derivatives (AlkSq- and ArSq-alkyne, Fig. 4c) also react with amines under physiological conditions. ${ }^{47}$ Both probes demonstrated very high selectivity for lysines (93\% and 90\%, Fig. 4b, Supplementary Fig. 23 and 24). Therefore, ArSq-alkyne is a promising broadly reactive alkyne probe for lysines (2990 quantified lysines), while structures like AlkSq-alkyne might be interesting for $\mathrm{TCl}$ design due to tempered reactivity (1339 quantified lysines). Another reactivity of lysines is the formation of imines with aldehydes, but irreversible stabilisation of the product is needed for the application as broadly reactive alkyne probe. Here, we were enticed by 2-ethynyl-benzaldehyde-based probes like EBA-alkyne (Fig. 4d) that were previously shown to react with amines in proteins to form an imine that cyclises to yield an irreversible isoquinolinium salt (Supplementary Fig. 25)..$^{48}$ Exhibiting high lysine selectivity (81\%) and good proteomic coverage (3796 quantified lysines, Fig. 4b, Supplementary Fig. 26 and 27), EBA-alkyne is an additional probe promising for lysine monitoring. Importantly, all of these probes also quantified a significant number of $\mathrm{N}$-termini (243 for AlkSq-alkyne, 252 for ArSq-alkyne and 253 for EBA-alkyne), which demonstrates monitoring of $\mathrm{N}$-termini as another possible application for these probes.

Light-activated probes hold the potential to monitor covalent target engagement in living cells as the probe itself is unreactive before activation and therefore has the potential to be non-toxic. ${ }^{10}$ Proteome-wide, residue-specific monitoring of lysines in a lightdependent fashion would therefore be highly promising to study effects on lysines with temporal and spatial resolution in living cells. Nevertheless, no probe had been previously shown to allow this 


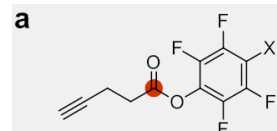

STP-alkyne $\mathrm{X}=\mathrm{SO}_{3} \mathrm{Na}$ TFP-alkyne $X=H$

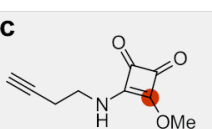

AlkSq-alkyne

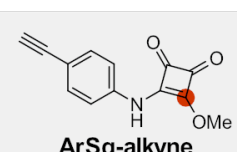

NHS-alkyne
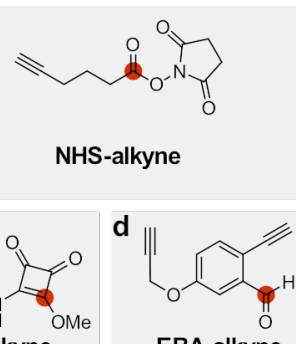

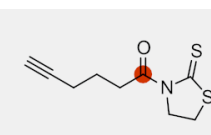

ATT-alkyne

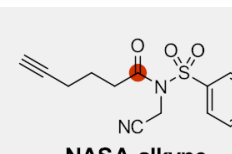

NASA-alkyne

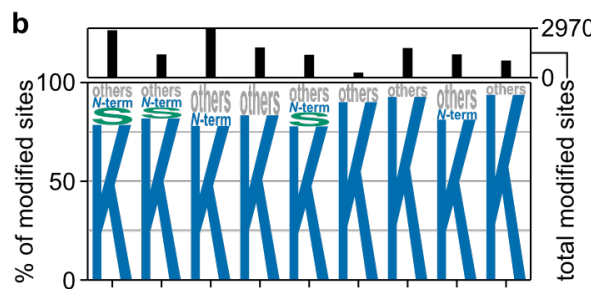

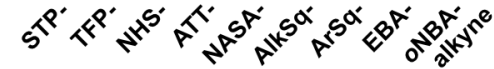

f

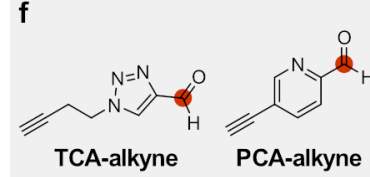

h

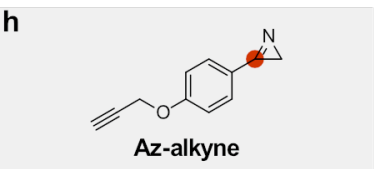

g
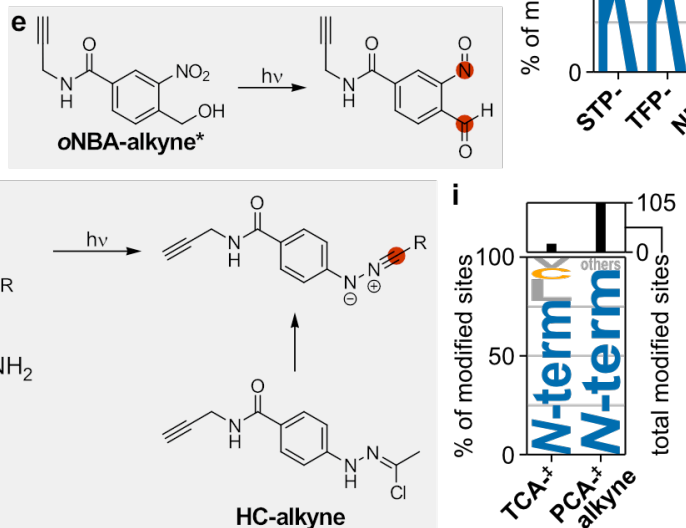

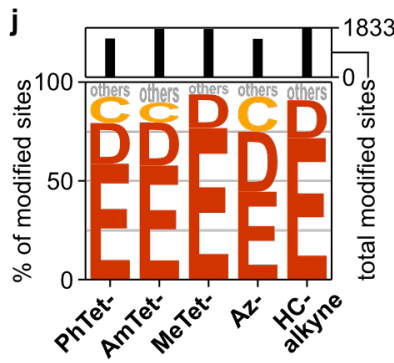

Fig. 4 | Amino acid selectivity of electrophiles targeting lysines, $\mathbf{N}$-termini, aspartates and glutamates. a,c,d,e,f,g,h, Structures of alkyne-containing electrophilic probes that were investigated for their proteome-wide amino acid selectivity. Red circles indicate the initial site of electrophilic reactivity. For probes oNBA-, PhTet-, AmTet-, MeTet- and HC-alkyne, the reactions leading to the reactive intermediate are also shown. $\mathbf{b}, \mathbf{i}, \mathbf{j}$, Amino acid selectivity of the probes upon treatment of the proteome of $\mathrm{S}$. aureus $\mathrm{SH} 1000$ at $100 \mu \mathrm{M}$ probe concentration. The data is represented as letter plots. The size of the letter is scaled by the fraction of all modified sites that is modified at the indicated amino acid. All amino acids that are modified in less than $5 \%$ of the cases are summarised as "others". The total number of modified sites is given as a bar graph on top of the letter plot. *: Labelling was performed using UV-activation at 280-315 nm (PhTet-, AmTet- and MeTet-alkyne) or $365 \mathrm{~nm}$ (oNBA-alkyne) for 10 min. $¥:$ : Data for the indicated probe at $1 \mathrm{mM}$ is shown. All data is based on technical duplicates.

application. For this purpose, we set out to study oNBA-alkyne that had been shown to react with lysines in live cells after formation of a nitrosobenzaldehyde upon irradiation (Fig. 4e, Supplementary Fig. 28). ${ }^{49}$ Indeed, we detected the expected modification (Supplementary Fig. 29 and 30) and a high degree of lysine selectivity (94\%) for this probe, which was retained even at $1 \mathrm{mM}$ probe concentration (93\%, Fig. 4b, Supplementary Fig. 29 and 30). Therefore, this is the first demonstration that oNBA-alkyne can act as a photoprobe to globally study lysines using residue-specific proteomics (1456 quantified lysines).

Taken the data of all lysine-directed probes together, we quantified 9129 lysines, which covers $15 \%$ of the 62,166 lysines encoded in the genome of $S$. aureus. Although STP-alkyne remains the reagent of choice to study lysines for standard applications, ArSq-, EBA- and oNBA-alkyne also display high selectivity using complementary chemistries. These probes will be important not only for increasing overall coverage (Supplementary Fig. 31), but also to allow specific applications, especially within living cells.

Global monitoring of $\mathbf{N}$-termini of proteins. Although several lysine-directed probes like STP-, ArSq- and EBA-alkyne also allow monitoring of $\mathrm{N}$-termini, selective chemistry is highly desirable. Here, carboxaldehydes of electron-poor heteroaromatics have been previously applied to modify proteins (TCA- ${ }^{50}$ and PCA-alkyne, ${ }^{51}$ Fig. 4f). While we were not able to detect more than a few sites for TCA-alkyne in the proteome even at $1 \mathrm{mM}$, PCA-alkyne resulted in the expected modification by initial formation of an imine with the $\mathrm{N}$-terminus followed by cyclisation involving the first amide nitrogen of the backbone (Supplementary Fig. 32 and 33). This modification showed high selectivity for the $\mathrm{N}$-terminus $(93 \%)$ and allowed quantification of $167 \mathrm{~N}$-termini at $1 \mathrm{mM}$ probe concentration (Fig. $4 \mathrm{i}$, Supplementary Fig. 33 and 34). Even though the coverage certainly still needs to be improved, PCA-alkyne is a suitable starting point to selectively monitor the $\mathrm{N}$-terminus proteome-wide. Taking the data of PCA- (1 mM), STP-, ArSq- and EBA-alkyne together, we were able to quantify $464 \mathrm{~N}$-termini in 412 proteins (some proteins were detected with and without clipping of the $\mathrm{N}$-terminal methionine) covering $14 \%$ of the 2959 proteins encoded in the S. aureus genome.

Monitoring aspartates and glutamates in the proteome. Recently, we have developed 2,5-disubstituted tetrazoles (Fig. 4g) into light-activatable tools to globally study aspartates and glutamates in living bacteria. ${ }^{10}$ Using our workflow, we verified the expected modification by formation of a nitrilimine upon light irradiation and subsequent reactivity eventually leading to an diacylated hydrazine (PhTet-, AmTet- and MeTet-alkyne, Fig. 4g, Supplementary Fig. 35 and 36). This modification showed a strong preference for aspartates and glutamates (79-94\%, Fig. 4j, Supplementary Fig. 36-38). The best probe, MeTet-alkyne, quantified 2192 aspartates and glutamates and its high selectivity was retained at $1 \mathrm{mM}$ probe concentration (93\%, Supplementary Fig. 36 and 38). While peptides labelled at aspartate were consistently enriched for aliphatic amino acids at position +1 (leucine, valine, isoleucine), those labelled at glutamate showed a preference for valine and alanine at positions -1 and -2 (Supplementary Fig. 37 and 38). ${ }^{43}$ When prolonging the photoreaction with MeTet-alkyne, we observed a decrease in the expected modification and an increasing additional modification that could be assigned as arylation, which localised to all aromatic amino acids and to cysteines (Supplementary Fig. 36 and 38). While this chemistry might therefore also be interesting to monitor aromatic amino acids, care must be taken to not overextend irradiation times when monitoring aspartates and glutamates with MeTet-alkyne.

$2 \mathrm{H}$-Azirines have been described as constitutively active electrophiles to target aspartates and glutamates by initial nucleophilic attack at the azirine eventually leading to an acylated $\alpha$-aminoketone (Az-alkyne, ${ }^{20}$ Fig. 4h, Supplementary Fig. 39). Using our workflow, we could show that besides the expected reactivity $\left(\Delta \mathrm{m}_{\exp }\right)$, these probes also showed a mass of modification with a mass of $\Delta \mathrm{m}_{\mathrm{exp}}+1 \mathrm{Da}$, which formally corresponds to hydrolysis leading to a loss of ammonia (Supplementary Fig. 39 and 40). As computationally differentiating these modifications was not fully possible, we further analysed their combined selectivity (Fig. $4 \mathrm{j}$, 

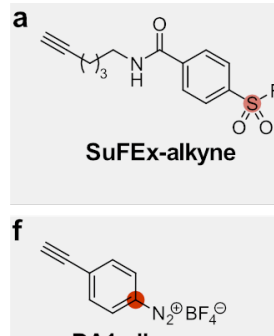

DA1-alkyne

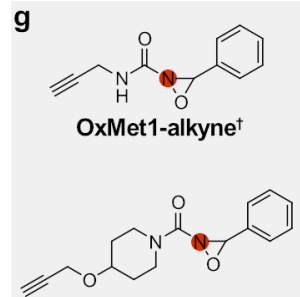

OxMet2-alkyne

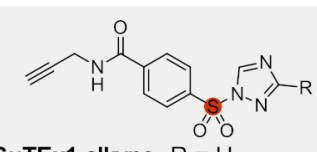

SuTEx1-alkyne $R=H$ SuTEx2-alkyne $\mathrm{R}=p-\mathrm{MeOC}_{6} \mathrm{H}_{4}$

b

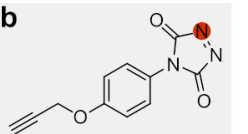
PTAD-alkyne

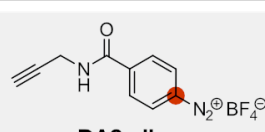

DA2-alkyne
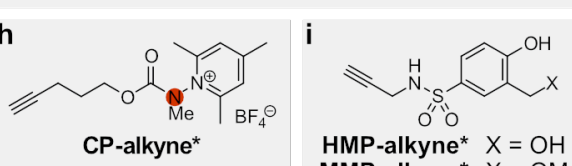

O'

HMP-alkyne ${ }^{*} \mathrm{X}=\mathrm{OH}$

MMP-alkyne ${ }^{*} X=\mathrm{OMe}$

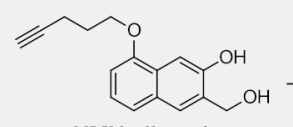

HMN-alkyne*
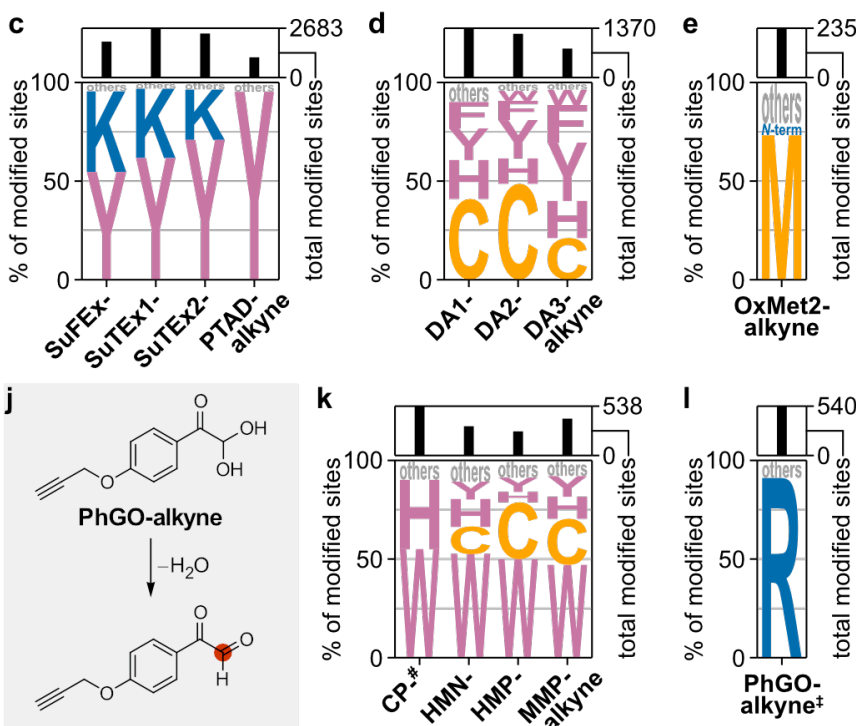

Fig. 5 | Amino acid selectivity of electrophiles targeting tyrosines, aromatic amino acids, methionines, tryptophans, histidines and arginines. a,b,f,g,h,i,j, Structures of alkyne-containing electrophilic probes that were investigated for their proteome-wide amino acid selectivity. Red circles indicate the initial site of electrophilic reactivity. For probes HMN-, HMP-, MMP- and PhGO-alkyne, the reactions leading to the reactive intermediate are also shown. $\mathbf{c}, \mathbf{d}, \mathbf{e}, \mathbf{k}, \mathbf{I}$, Amino acid selectivity of the probes upon treatment of the proteome of $\mathrm{S}$. aureus $\mathrm{SH} 1000$ at $100 \mu \mathrm{M}$ probe concentration. The data is represented as letter plots. The size of the letter is scaled by the fraction of all modified sites that is modified at the indicated amino acid. All amino acids that are modified in less than $5 \%$ of the cases are summarised as "others". The total number of modified sites is given as a bar graph on top of the letter plot. t: No clear mass of modification was detected and therefore no analysis of the amino acid selectivity was possible. *: Labelling

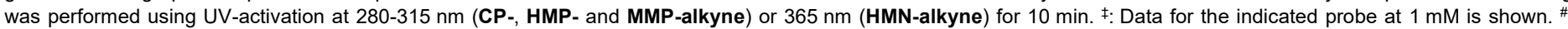
Labelling was performed in degassed lysate under argon. All data is based on technical duplicates.

Supplementary Fig. 40 and 41) and identified $75 \%$ of all modifications at aspartates and glutamates and $18 \%$ at cysteines. Az-alkyne is therefore a valuable probe to study aspartates and glutamates, but care must be taken to account for cysteine off-targets.

Enticed by the high selectivity of MeTet-alkyne, we also explored similar chemistry for monitoring aspartates and glutamates with constitutively active electrophiles. Hydrazonoyl chlorides such as HC-alkyne (Fig. $4 \mathrm{~g}$ ) have been shown to liberate the same reactive nitrilimine as the light-activated tetrazoles (Supplementary Fig. 35 ). ${ }^{52}$ We detected the expected modification and high selectivity for aspartates and glutamates (91\%), which was also retained at $1 \mathrm{mM}$ probe concentration (92\%, Fig. 4j, Supplementary Fig. 42 and 43). The sequence around the labelled amino acids showed the same preferences as also seen for MeTet-alkyne (Supplementary Fig. 43). ${ }^{43} \mathrm{HC}$-alkyne allowed the quantification of 2450 aspartates and glutamates in the proteome at $100 \mu \mathrm{M}$ and is therefore a novel promising probe to study these amino acids without the need for light-activation.

Interestingly, MeTet- and $\mathbf{H C}$-alkyne showed a strong preference to label glutamates $(77 \%$ and $71 \%)$ over aspartates $(17 \%$ and $19 \%)$, while Az-alkyne demonstrated a smaller difference $(44 \%$ and $30 \%$, respectively) indicating that it is better able to also react with the sterically more hindered aspartate. Taking all carboxylic aciddirected probes together, 7811 aspartates and glutamates were quantified corresponding to $7.8 \%$ of the 100,780 aspartates and glutamates encoded in the S. aureus genome. Specifically, MeTet-, HC- and Az-alkyne constitute a set of complementary probes that allow a deep profiling of these amino acids in the bacterial proteome (Supplementary Fig. 44). Considering that no probes exist to selectively monitor $C$-termini proteome-wide, it is furthermore noteworthy that all carboxylic acid-directed probes together quantified $179 \mathrm{C}$-termini with MeTet- and $\mathrm{HC}$-alkyne at $1 \mathrm{mM}$ being most promising (101 and 109 quantified $C$-termini).
Residue-specific proteomics at tyrosines. Tyrosines offer a unique opportunity for various selective chemistries through reactions with the hydroxyl group as well as with the electron-rich aromatic system. Recently, sulfonylation of the hydroxyl group using sulfur-fluoride (SuFEx-alkyne $)^{53}$ and sulfur-triazole exchange chemistry (SuTEx1- and SuTEx2-alkyne) ${ }^{23,24}$ has been established for proteome-wide approaches (Fig. 5a). We verified the tyrosine reactivity of these probes $(55-71 \%$, Fig. 5 c, Supplementary Fig. 45 and 46) with lysine residues being the most prominent off-targets (26-41\%). In agreement with a previous study in human proteomes, SuTEx2-alkyne showed the highest tyrosine selectivity and allowed quantifying 2653 tyrosines in bacterial lysates.

For labelling of the aromatic system of tyrosines in proteins, reagents like PTAD-alkyne have been established (Fig. 5b, Supplementary Fig. 47). ${ }^{54}$ We identified the expected adduct that shows high selectivity for tyrosines (95\%, Fig. 5c, Supplementary Fig. 48 and 49). Furthermore, we also detected a modification that corresponds to fragmentation of PTAD-alkyne to the isocyanate and subsequent reactivity, which showed some selectivity for lysines and $\mathrm{N}$-termini and could be strongly reduced through addition of excess primary amine (Supplementary Fig. 48 and 49). ${ }^{55}$ Due to the exquisite selectivity of the expected modification, it would be interesting to optimise the stability of these reagents to further reduce this side reactivity.

Through combination of the data for all tyrosine probes, we quantified 3968 tyrosines covering $12 \%$ of the 32,172 tyrosines encoded in the $S$. aureus genome. While SuTEx2-alkyne is currently the probe of choice for tyrosines, reagents like PTAD-alkyne should allow for the development of optimised complementary probes in the future (Supplementary Fig. 50).

Diazonium salts perform arylation chemistry in the bacterial proteome. During the investigation of tyrosine-directed chemistries, we also considered aryl diazonium salts that have been shown to lead to azo coupling on tyrosines in isolated proteins (Fig. $5 f$, Supplementary Fig. 51). ${ }^{56}$ Proteome-wide, we only detected minor 


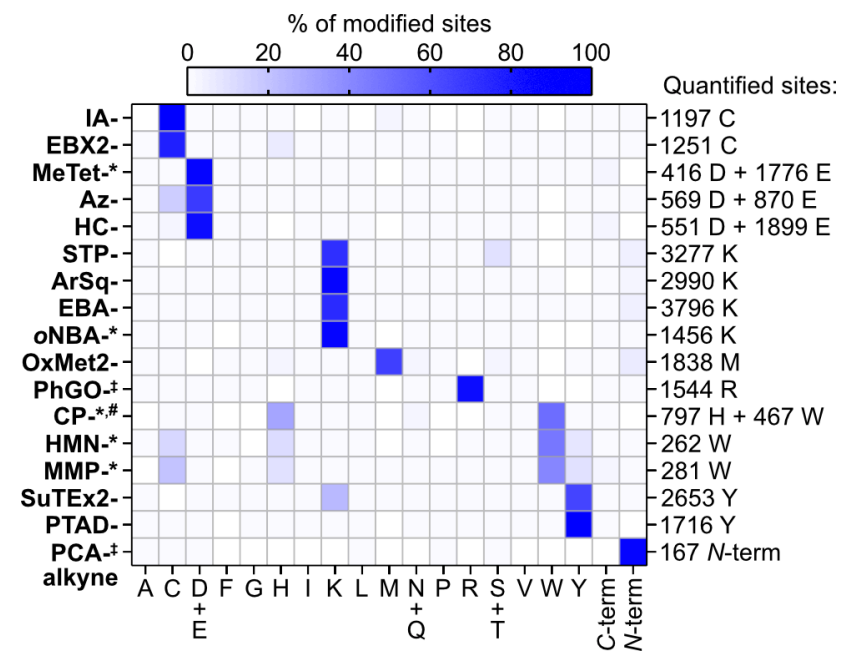

Fig. 6 | A set of 17 electrophilic probes that enables studying nine different amino acid residues and the $\mathbf{N}$-terminus. For a selection of the probes that allow studying diverse residues in the proteome of $S$. aureus, their selectivity is plotted in a heatmap. The colour is scaled by the fraction of all modified sites that is modified at the indicated amino acid. *: Labelling was performed using UV-activation at 280-315 nm (MeTet-, CP- and MMP-alkyne) or 365 nm (oNBA- and HMN-alkyne) for $10 \mathrm{~min}$. ¥: Data for the indicated probe at $1 \mathrm{mM}$ is shown. \#: Labelling was performed in degassed lysate under argon. All data is based on technical duplicates.

azo coupling and almost exclusively arylation, corresponding to a formal loss of molecular nitrogen (Supplementary Fig. 51-53). ${ }^{57}$ Strikingly, next to modifications on cysteines, ${ }^{58}$ this also led to up to $75 \%$ of all modifications being localised to aromatic amino acids for DA3-alkyne (19\% phenylalanines, $19 \%$ histidines, $8 \%$ tryptophans, $30 \%$ tyrosines, Fig. 5d, Supplementary Fig. 52 and 53). In this way, DA3-alkyne enabled the quantification of 1218 aromatic amino acids. While aryl diazonium salts are therefore not suitable for azo couplings in bacterial proteomes, it is noteworthy that they can be used to monitor various aromatic amino acids and that preferred reactivity is possible at many residues in the proteome including even the unactivated aromatic system of phenylalanines.

An optimised tool for global monitoring of methionine residues. Hypervalent iodine reagents have been described to monitor methionines but require an additional reaction step to give a stable modification. ${ }^{22}$ Therefore, we focused on oxaziridines for labelling of methionines, which proceeds through initial attack of the thioether at the nitrogen of the oxaziridine eventually giving a sulfimide (Supplementary Fig. 54). ${ }^{21}$ The initially described reagent OxMet1-alkyne (Fig. $5 \mathrm{~g}$ ) did not result in detection of the expected modification using our standard workflow (Supplementary Fig. 55). Implementing recently described design principles for more stable methionine modification, ${ }^{59}$ we synthesised OxMet2-alkyne (Fig. $5 \mathrm{~g}$ ), which led to the detection of a high number of modified peptides with a preference for methionine modification $(73 \%, 1838$ quantified methionines, $8.5 \%$ of the 21,677 methionines encoded in the S. aureus genome, Fig. 5e, Supplementary Fig. 55 and 56). In this way, we developed OxMet2-alkyne as a tailored reagent for proteome-wide monitoring of methionines.

The first technologies to monitor tryptophans and histidines proteome-wide. Recently, $\mathrm{N}$-carbamoylpyridinium salts like CP-alkyne (Fig. 5h) have been introduced to photochemically label tryptophans in proteins through photoinduced electron transfer leading to the attachment of the carbamoyl group to tryptophans (Supplementary Fig. 57). ${ }^{60}$ However, proteome-wide studies were still missing. Upon irradiation under protective gas, CP-alkyne showed the expected mass of modification in the proteome with almost complete selectivity for tryptophans (55\%) and histidines (35\%, Fig. 5k, Supplementary Fig. 58 and 59) allowing the quantification of 467 tryptophans and 797 histidines. The selectivity was retained, when the reaction was run open to air or at $1 \mathrm{mM}$ CP-alkyne (Supplementary Fig. 58 and 59). While we could not detect any preferences in the sequence around labelled histidine, peptides modified at tryptophan consistently showed an enrichment of glutamate in the -1-position (Supplementary Fig. 59). ${ }^{43}$ The reactivity with histidines is especially noteworthy, as we were unsuccessful to detect the expected modification with reported histidine-selective thiophosphorodichloridates (TPAC-alkyne, Supplementary Fig. 60), most likely due to instability of the conjugate during our workflow. ${ }^{61}$ The fraction of $>35 \%$ of histidine labelling for CP-alkyne is the highest we have detected for any probe, making it the probe of choice to study histidines proteome-wide. Taking all conditions for CP-alkyne together, we quantified 1697 histidines covering $9.1 \%$ of the 18,746 histidines in the $S$. aureus genome. CP-alkyne is, therefore, the first probe to globally monitor both tryptophans and histidines using residue-specific proteomics.

Simultaneously, we also investigated UV-activatable o-quinone methide precursors for tryptophan labelling through a formal [4+2]-cycloaddition (HMN-, HMP- and MMP-alkyne, Fig. 5i, Supplementary Fig. 61). Also in this case, we observed a preference for tryptophans (47-53\%, Fig. 5k, Supplementary Fig. 62 and 63 ) with cysteines $(14-29 \%)$, histidines $(5-13 \%)$ and tyrosines $(7-10 \%)$ being the main off-targets. In this way, HMN- and MMP-alkyne are complementary probes to study tryptophans in a proteome-wide sense. Through the combination of the data for all probes, we quantified a total of 701 tryptophans covering $11 \%$ of the 6183 tryptophans encoded in the $S$. aureus genome (Supplementary Fig. 64).

A first-in-class method to monitor arginines in the whole proteome. Although arginine has been targeted with glyoxal-based reagents for bioconjugation ${ }^{62}$ and crosslinking before, ${ }^{63}$ no method is currently available to globally monitor arginines with residue-specific proteomics. We therefore synthesised PhGO-alkyne based on the known reactivity of phenylglyoxals with arginines that eventually produces a stable imidazole derivative (Fig. 5 j, Supplementary Fig. 65). Using our workflow, we detected only minor modification of the proteome with the expected modification accompanied by an oxidation product (Supplementary Fig. 66). ${ }^{64}$ At increased concentration of $1 \mathrm{mM}$ PhGO-alkyne, however, we detected many modifications of the proteome (1544 arginines quantified, $5.4 \%$ of the 28,550 arginines encoded in the $S$. aureus genome) with the expected mass and high arginine selectivity (91\%) alongside some oxidation (Fig. 5l, Supplementary Fig. 66 and 67). While the presence of the oxidation product still necessitates some optimisation, PhGo-alkyne is the first probe to globally monitor arginine in the proteome.

A set of probes to globally study nine amino acids and the $\boldsymbol{N}$-terminus. Through screening of 54 alkyne probes, we have identified a set of 17 probes that we currently consider ideal for profiling nine different amino acids and the $\mathrm{N}$-terminus proteomewide (Fig. 6). The fraction of all residues of a certain amino acid that we quantified using this probe set ranged from $2.5 \%$ of all aspartates to $29 \%$ of all cysteines encoded in the genome of $S$. aureus. In total, we were able to quantify 20,558 different sites in the proteome using our probe selection. These sites cover 1399 of 2959 proteins encoded in the S. aureus genome (54\%) and $85 \%$ of the annotated essential proteins (301/353). ${ }^{65}$ In this way, our probe selection allows us to gain very deep insights into the bacterial proteome. 
To show that the application of our probe selection is not restricted to bacterial systems, we also applied these probes in lysates of the human cancer cell line MDA-MB-231. Except for PCA-alkyne, we reproduced similar selectivities for all probes in the human proteome (Supplementary Fig. 68-76). In this way, we were able to quantify a total of 23,831 sites in 3770 proteins in this human cell line.

\section{Conclusion}

We report here on a universal, unbiased workflow to study the reactivity and selectivity of electrophilic probes in a proteome-wide setup. For this purpose, we extended and established new components of the broadly available MSFragger-based FragPipe computational platform. ${ }^{6,7}$ These include an optimised workflow for filtering of Open Searches to reliably identify masses of modification, a new workflow for downstream data analysis of Offset Searches to investigate selectivity at all proteogenic amino acids simultaneously and a new feature for lonQuant that now allows to quantify data from stable isotopic labelling-based MS experiments. In this way, we are for the first time able to identify the added masses of modification, their selectivity at all proteinogenic amino acids and their relative abundance in a completely unbiased fashion.

While our selection of 54 alkyne probes is certainly not comprehensive, we are convinced that we covered most chemistries that have more broadly been applied for protein labelling and that do not require additional reagents. In this way, we verified and newly identified tailored probes to study nine different amino acids as well as the $\mathrm{N}$-terminus proteome-wide. Within our set of probes, we verify the selectivity of IA- and EBX2-alkyne for cysteines, ${ }^{2,3}$ STP-alkyne for lysines, ${ }^{18}$ SuTEx2-alkyne for tyrosines ${ }^{23}$ as well as MeTet- ${ }^{10}$ and Az-alkyne ${ }^{20}$ for aspartates and glutamates. For several of these amino acids, we identify additional probes based on complementary chemistries like ArSq- and EBA-alkyne for lysines, HC-alkyne for aspartates and glutamates and PTAD-alkyne for tyrosines, which increase the proteome-wide coverage. For lysines, we demonstrate for the first time that proteome-wide residue-specific profiling is possible in a light-dependent fashion using oNBA-alkyne, which opens up exciting opportunities for in cell studies. Additionally, we developed a tailored probe (OxMet2-alkyne) for methionines that allows application in our workflow with high coverage due to increased stability of the protein modification. Furthermore, we transferred the concept of labelling proteins at the $\mathrm{N}$-terminus to global residue-specific studies using PCA-alkyne. Strikingly, our study has enabled us to develop probes that for the first time allow studying tryptophans (CP-, HMN-, MMP-alkyne), histidines (CP-alkyne) and arginines (PhGO-alkyne) in a residue-specific fashion in the whole proteome.

Important residues, for which no selective broadly reactive alkyne probes could be established here, are serines and threonines as well as $C$-termini. For serines and threonines, bioconjugation to proteins using $\mathrm{P}(\mathrm{V})$-based reagents for phosphorus-sulfur incorporation (PSI) has recently been established. ${ }^{66,67}$ Nevertheless, we were unable to detect a clear modification for PSI-alkyne (Supplementary Fig. 77 and 78), most likely due to instability of the conjugate during our workflow. In our hands, STP-alkyne is still the best way to monitor at least some serines and threonines in a residue-specific fashion. For $C$-termini, the aspartate- and glutamate-directed probes allowed us to quantify $179 \mathrm{C}$-termini, but increased coverage and selective probes are surely desirable here.

One important application of our probe selection will be to competitively study target engagement for any covalently reactive protein ligand regardless of its amino acid selectivity and adduct stability. This will be especially important for ligands, for which probe synthesis is challenging or which form covalent adducts that evade direct detection like those forming unstable modifications such as thioesters ${ }^{13}$ or covalently reversible protein adducts. ${ }^{14,15}$ It is tempting to speculate that in the future it will be possible to use a mixture of several probes to competitively profile the selectivity of a protein ligand simultaneously at various amino acids within the same proteomic experiment as has recently been described for the combined detection of cysteines and lysines. ${ }^{13}$

Taking all of this together, we report on an unbiased, universal workflow to characterise amino acid selectivity of electrophilic compounds in the whole proteome. These studies have enabled the profiling of established, tailored as well as new probes for residuespecific proteomics, which now allows monitoring of a total of nine different amino acids and the $\mathrm{N}$-terminus. We are convinced that our workflow and this probe selection will be instrumental to identifying selectively reactive groups for the design of covalent inhibitors targeting diverse amino acids. In this way, they will advance the development of $\mathrm{TCls}$ for the many protein binding sites that lack a suitable cysteine residue for engagement with the currently dominant acrylamide-based chemistry.

\section{Data availability}

Mass spectrometric data for all proteomic analyses have been deposited to the ProteomeXchange Consortium (http://proteomecentral.proteomexchange.org) via the PRIDE partner repository ${ }^{68}$ with the dataset identifier PXD024454. Raw data for Fig. 2-6 is available in Supplementary Tables 1-4. All other data is available in the main text or the Supplementary Information.

\section{Code Availability}

Code for the MSFragger-based FragPipe analyses is available at https://fragpipe.nesvilab.org/.

\section{Acknowledgements}

S.M.H. and P.R.A.Z. acknowledge funding by the Fonds der Chemischen Industrie through a Liebig Fellowship and a Ph.D. fellowship and by the TUM Junior Fellow Fund. A.I.N. acknowledges financial support from the US National Institutes of Health (R01-GM-094231 and U24-CA210967). F.D.T. thanks the Novartis Institutes for BioMedical Research and the NovartisBerkeley Center for Proteomics and Chemistry Technologies for supporting this work. P.Z.M. thanks the American Cancer Society for a post-doctoral fellowship (PF-18-132-01-CDD). M.Z. gratefully acknowledges funding by the Studienstiftung des Deutschen Volkes through a Ph.D. fellowship. K.L. is grateful for support by the collaborative research centre SFB1035 (German Research Foundation DFG, Sonderforschungsbereich 1035, Projektnummer 201302640, project B10). C.J.C. acknowledges funding by the NIH (ES 28096 and GM139245). We gratefully acknowledge Stephan A. Sieber and his group for their generous support. We thank the group of Jérôme Waser (EPFL) for providing EBX1-alkyne, Ku-Lung Hsu and Jeffrey W. Brulet (University of Virginia) for providing SuTEx1-alkyne and SuTEX2-alkyne and the group of Phil S. Baran (Scripps Research) for providing PSI-alkyne. We thank Simon Kollmannsberger and Maximilian Iglhaut (Technical University of Munich) for assisting with the synthesis of probes as well as Katja Bäuml and Mona Wolff (Technical University of Munich) for technical assistance. We thank Daniel Geiszler, Felipe da Veiga Leprevost and Dmitry Avtonomov for useful discussions and technical assistance regarding new developments in FragPipe. 


\section{Author Contributions}

P.R.A.Z. and S.M.H. designed the research, planned experiments, performed and analysed proteomics experiments. F.Y. and A.I.N. designed, developed and benchmarked data analysis software. P.R.A.Z. synthesised the majority of probes. P.Z.M., L.L., M.Z., K.K., D.M., P.R., T.E.M., and M.C. contributed the synthesis of individual probes. K.L., C.J.C. and F.D.T. designed individual probes. P.R.A.Z. and S.M.H. wrote the manuscript with input from all authors.

\section{Competing interests}

The authors declare no competing interests.

\section{Additional information}

Supplementary information is available for this paper in the online version.

Correspondence and requests for materials should be addressed to S.M.H.

\section{References}

Singh, J., Petter, R. C., Baillie, T. A. \& Whitty, A. The resurgence of covalent drugs. Nat. Rev. Drug. Discov. 10, 307-317 (2011). Backus, K. M. et al. Proteome-wide covalent ligand discovery in native biological systems. Nature 534, 570-574 (2016).

Weerapana, E. et al. Quantitative reactivity profiling predicts functional Zanon, P. R. A., Lewald, L. \& Hacker, S. M. Isotopically Labeled Desthiobiotin Azide (isoDTB) Tags Enable Global Profiling of the Bacterial Cysteinome. Angew. Chem. Int. Ed., 2829-2836 (2020). Rostovtsev, V. V., Green, L. G., Fokin, V. V. \& Sharpless, K. B. A stepwise huisgen cycloaddition process: copper(I)-catalyzed regioselective "ligation" of azides and terminal alkynes. Angew. Chem. Int. Ed. 41, 2596-2599 (2002).

Kong, A. T., Leprevost, F. V., Avtonomov, D. M., Mellacheruvu, D. \& Nesvizhskii, A. I. MSFragger: ultrafast and comprehensive peptide identification in mass spectrometry-based proteomics. Nat. Methods 14, 513-520 (2017).

$\mathrm{Yu}, \mathrm{F}$. et al. Identification of modified peptides using localization-aware open search. Nat. Commun. 11, 4065 (2020).

Yu, F. et al. Fast Quantitative Analysis of timsTOF PASEF Data with MSFragger and IonQuant. Mol. Cell.Proteom. 19, 1575-1585 (2020). Sutanto, F., Konstantinidou, M. \& Dömling, A. Covalent inhibitors: a rational approach to drug discovery. RSC Med. Chem. 11, 876-884 (2020)

Bach, K., Beerkens, B. L. H., Zanon, P. R. A. \& Hacker, S. M. LightActivatable, 2,5-Disubstituted Tetrazoles for the Proteome-Wide Profiling of Aspartates and Glutamates in Living Bacteria. ACS Cent. Sci. 6, 546-554 (2020).

Gehringer, M. \& Laufer, S. A. Emerging and Re-Emerging Warheads for Targeted Covalent Inhibitors: Applications in Medicinal Chemistry and Chemical Biology. J. Med. Chem. 62, 5673-5724 (2019).

Parker, C. G. \& Pratt, M. R. Click Chemistry in Proteomic Investigations. Cell 180, 605-632 (2020)

Cao, J. et al. Multiplexed CuAAC Suzuki-Miyaura Labeling for Tandem Activity-Based Chemoproteomic Profiling. Anal. Chem. 93, 2610-2618 (2021)

Lee, K. M., Le, P., Sieber, S. A. \& Hacker, S. M. Degrasyn exhibits antibiotic activity against multi-resistant Staphylococcus aureus by modifying several essential cysteines. Chem. Commun. 56, 2929-2932 (2020).

Senkane, K. et al. The Proteome-Wide Potential for Reversible (2019).

Motiwala, H. F., Kuo, Y.-H., Stinger, B. L., Palfey, B. A. \& Martin, B. R. Tunable Heteroaromatic Sulfones Enhance in-Cell Cysteine Profiling. J. Am. Chem. Soc. 142, 1801-1810 (2020)

Abegg, D. et al. Proteome-Wide Profiling of Targets of Cysteine reactive Small Molecules by Using Ethynyl Benziodoxolone Reagents. Angew. Chem. Int. Ed. 54, 10852-10857 (2015).

Hacker, S. M. et al. Global profiling of lysine reactivity and ligandability in the human proteome. Nat. Chem. 9, 1181-1190 (2017).

Ward, C. C., Kleinman, J. I. \& Nomura, D. K. NHS-Esters As Versatile Reactivity-Based Probes for Mapping Proteome-Wide Ligandable Hotspots. ACS Chem. Biol. 12, 1478-1483 (2017). cysteines in proteomes. Nature 468, 790-795 (2010). Covalency at Cysteine. Angew. Chem. Int. Ed. 58, 11385-11389
Soc. 142 6051-6059 (2020).

Lin, S. et al. Redox-based reagents for chemoselective methionine bioconjugation. Science 355, 597-602 (2017).

Taylor, M. T., Nelson, J. E., Suero, M. G. \& Gaunt, M. J. A protein functionalization platform based on selective reactions at methionine residues. Nature 562, 563-568 (2018).

Hahm, H. S. et al. Global targeting of functional tyrosines using sulfurtriazole exchange chemistry. Nat. Chem. Biol. 16, 150-159 (2019). Brulet, J. W., Borne, A. L., Yuan, K., Libby, A. H. \& Hsu, K.-L. Liganding Functional Tyrosine Sites on Proteins Using Sulfur-Triazole Exchange Chemistry. J. Am. Chem. Soc. 142, 8270-8280 (2020). Weerapana, E., Simon, G. M. \& Cravatt, B. F. Disparate proteome reactivity profiles of carbon electrophiles. Nat. Chem. Biol. 4, 405-407 (2008).

Horsburgh, M. J. et al. sigmaB modulates virulence determinan expression and stress resistance: characterization of a functional rsbU strain derived from Staphylococcus aureus 8325-4. J. Bacteriol. 184 5457-5467 (2002).

Benns, H. J., Wincott, C. J., Tate, E. W. \& Child, M. A. Activity- and reactivity-based proteomics: Recent technological advances and applications in drug discovery. Curr. Opin. Chem. Biol. 60, 20-29 (2021). Open Search Results. J. Proteome Res. 19, 2511-2515 (2020). Geiszler, D. J. et al. PTM-Shepherd: analysis and summarization of post-translational and chemical modifications from open search results. Mol. Cell. Proteom. (2020).

Keller, A., Nesvizhskii, A. I., Kolker, E. \& Aebersold, R. Empirical statistical model to estimate the accuracy of peptide identifications made by MS/MS and database search. Anal. Chem. 74, 5383-5392 (2002).

. Keller, A Kolker E \& Aebersold, R A statistical model for identifying proteins by tandem mass spectrometry. Anal. Chem. 75, 4646-4658 (2003). . shotgun proteomics data analysis. Nat. Methods 17, 869-870 (2020). Lenčo, J., Khalikova, M. A. \& Švec, F. Dissolving Peptides in $0.1 \%$ Formic Acid Brings Risk of Artificial Formylation. J. Proteome Res. 19 993-999 (2020) . Proteome Res. 20, 498-505 (2021). redox and electrophile reactive cysteine proteomes. Nat. Protoc. $\mathbf{1 5}$ 2891-2919 (2020).

Cox, J. \& Mann, M. MaxQuant enables high peptide identification rates, individualized p.p.b.-range mass accuracies and proteome-wide protein quantification. Nat. Biotechnol. 26, 1367-1372 (2008). Chi, H. et al. Comprehensive identification of peptides in tandem mass spectra using an efficient open search engine. Nat. Biotechnol. 36 1059-1061 (2018).

Abo, M. \& Weerapana, E. A Caged Electrophilic Probe for Global Analysis of Cysteine Reactivity in Living Cells. J. Am. Chem. Soc. 137 7087-7090 (2015).

Embaby, A. M. Schoffelen, S., Kofoed, C., Meldal, M. \& Diness, F. Rational Tuning of Fluorobenzene Probes for Cysteine-Selective Protein Modification. Angew. Chem. Int. Ed. 57, 8022-8026 (2018). R. et al. Fast and Highly Chemoselective Alkynylation of Thiols with Hypervalent lodine Reagents Enabled through a Low Energy Barrier Concerted Mechanism. J. Am. Chem. Soc. 136, 16563-16573 (2014)

(2) ablides and Proteins. Chem 5, 2243-2263 (2019).

Tessier, R. et al. Ethynylation of Cysteine Residues: From Peptides to Proteins in Vitro and in Living Cells. Angew. Chem. Int. Ed. 59, 1096110970 (2020)

Shea, J. P. et al. pLogo: a probabilistic approach to visualizing sequence motifs. Nat. Methods. 10, 1211-1212 (2013). Pettinger, J., Jones, K. \& Cheeseman, M. D. Lysine-Targeting Covalent Inhibitors. Angew. Chem. Int. Ed. 56, 15200-15209 (2017). Hirata, T. et al. Synthesis and reactivities of 3-indocyanine-green-acyl1,3-thiazolidine-2-thione (ICG-ATT) as a new near-infrared fluorescent-labeling reagent. Bioorg. Med. Chem. 6, 2179-2184 (1998)

Tamura, T. et al. Rapid labelling and covalent inhibition of intracellular native proteins using ligand-directed $\mathrm{N}$-acyl-N-alkyl sulfonamide. Nat. Commun. 9, 1870 (2018)

Ivancová, I., Pohl, R., Hubálek, M. \& Hocek, M. Squaramate-Modified Nucleotides and DNA for Specific Cross-Linking with LysineContaining Peptides and Proteins. Angew. Chem. Int. Ed. 58, 1334513348 (2019)

Deng, J.-R. et al. N-Terminal selective modification of peptides and proteins using 2-ethynylbenzaldehydes. Commun. Chem. 3, 67 (2020)

A.-D. et al. Light-induced primary amines and o-nitrobenzy alcohols cyclization as a versatile photoclick reaction for modular conjugation. Nat. Commun. 11, 5472 (2020). 

site-specific modification of native proteins with 2

pyridinecarboxyaldehydes. Nat. Chem. Bio. 11, 326-331 (2015). Zengeya, T. T. et al. Co-opting a Bioorthogonal Reaction for Oncometabolite Detection. J. Am. Chem. Soc. 138, 15813-15816 (2016)

53 Dong, J., Krasnova, L., Finn, M. G. \& Sharpless, K. B. Sulfur(VI) Fluoride Exchange (SuFEx): Another Good Reaction for Click Chemistry. Angew. Chem. Int. Ed. 53, 9430-9448 (2014). Ban, H., Gavrilyuk, J. \& Barbas, C. F. Tyrosine bioconjugation through aqueous ene-type reactions: a click-like reaction for tyrosine. J. Am. Chem. Soc. 132, 1523-1525 (2010).

55 Ban, H. et al. Facile and Stabile Linkages through Tyrosine: Bioconjugation Strategies with the Tyrosine-Click Reaction. Bioconjug. Chem. 24, 520-532 (2013).

56 Zhang, J., Ma, D., Du, D., Xi, Z. \& Yi, L. An efficient reagent for covalent introduction of alkynes into proteins. Org. Biomol. Chem. 12 9528-9531 (2014).

57 Nothling, M. D. et al. Bacterial Redox Potential Powers Controlled Radical Polymerization. J. Am. Chem. Soc. 143, 286-293 (2021).

Naveen, N., Sengupta, S. \& Chandrasekaran, S. Metal-Free SArylation of Cysteine Using Arenediazonium Salts. J. Org. Chem. 83 3562-3569 (2018).

59 Christian, A. H. et al. A Physical Organic Approach to Tuning Reagents for Selective and Stable Methionine Bioconjugation. J. Am. Chem. Soc. 141, 12657-12662 (2019).

60 Tower, S. J., Hetcher, W. J., Myers, T. E., Kuehl, N. J. \& Taylor, M. T. Selective Modification of Tryptophan Residues in Peptides and Proteins Using a Biomimetic Electron Transfer Process. J. Am. Chem. Soc. 142, 9112-9118 (2020).

61 Jia, S., He, D. \& Chang, C. J. Bioinspired Thiophosphorodichloridate Reagents for Chemoselective Histidine Bioconjugation. J. Am. Chem. Soc. 141, 7294-7301 (2019).

62 Dovgan, I. et al. Arginine-selective bioconjugation with 4-azidophenyl glyoxal: application to the single and dual functionalisation of native antibodies. Org. Biomol. Chem. 16, 1305-1311 (2018).

63 Jones, A. X. et al. Improving mass spectrometry analysis of protein structures with arginine-selective chemical cross-linkers. Nat. Commun. 10, 3911 (2019)

Thompson, D. A., Ng, R. \& Dawson, P. E. Arginine selective reagents for ligation to peptides and proteins. J. Pept. Sci. 22, 311-319 (2016). Chaudhuri, R. R. et al. Comprehensive identification of essential

Staphylococcus aureus genes using Transposon-Mediated Differential Hybridisation (TMDH). BMC Genomics 10, 291 (2009). Vantourout, J. C. et al. Serine-Selective Bioconjugation. J. Am. Chem. Soc. 142, 17236-17242 (2020)

$67 \quad$ Knouse, K. W. et al. Unlocking P(V): Reagents for chiral phosphorothioate synthesis. Science 361, 1234-1238 (2018)

68 Perez-Riverol, Y. et al. The PRIDE database and related tools and resources in 2019: improving support for quantification data. Nucleic Acids Res. 47, D442-d450 (2019). 